\title{
Deubiquitinase USP5 promotes non-small cell lung cancer cell proliferation by stabilizing cyclin D1
}

\author{
Zhiwei Zhang ${ }^{1 \# \wedge}$, Zihan Cui ${ }^{1 \#}$, Zhuolin Xie ${ }^{1}$, Chang $\mathrm{Li}^{1}$, Chun $\mathrm{Xu}^{1}$, Xia Guo ${ }^{2}$, Jie Yu ${ }^{2}$, Tengfei Chen ${ }^{1}$, \\ Francesco Facchinetti ${ }^{3}$, Hanibal Bohnenberger ${ }^{4}$, Tracy L. Leong ${ }^{5}$, Yufeng Xie ${ }^{6}$, Xinliang Mao ${ }^{7,8}$, Jun Zhao ${ }^{1}$ \\ ${ }^{1}$ Department of Thoracic Surgery, the First Affiliated Hospital of Soochow University, Suzhou, China; ${ }^{2}$ Department of Pathology, the First Affiliated \\ Hospital of Soochow University, Suzhou, China; ${ }^{3}$ Université Paris-Saclay, Institut Gustave Roussy, Inserm, Biomarqueurs Prédictifs et Nouvelles, \\ Stratégies Thérapeutiques en Oncologie, Villejuif, France; ${ }^{4}$ Institute of Pathology, University Medical Center, Göttingen, Germany; ${ }^{5}$ Department of \\ Respiratory Medicine, Austin Health, Heidelberg, Victoria, Australia; ${ }^{6}$ Department of Oncology, the First Affiliated Hospital of Soochow University, \\ Suzhou, China; ${ }^{7}$ Department of Pharmacology, College of Pharmaceutical Sciences, Soochow University, Suzhou, China; ${ }^{8}$ Guangdong Key \\ Laboratory of Protein Modification and Degradation, School of Basic Medical Sciences, Guangzhou Medical University, Guangzhou, China \\ Contributions: (I) Conception and design: J Zhao, X Mao; (II) Administrative support: J Zhao, X Mao, Y Xie; (III) Provision of study materials or \\ patients: Z Zhang, Z Cui, C Xu, C Li; (IV) Collection and assembly of data: Z Zhang, Y Xie, J Zhao; (V) Data analysis and interpretation: Z Zhang, \\ Y Xie; (VI) Manuscript writing: All authors; (VII) Final approval of manuscript: All authors. \\ "These authors contributed equally to this work. \\ Correspondence to: Dr. Jun Zhao. Department of Thoracic Surgery, the First Affiliated Hospital of Soochow University, 188 Shizi Street, Suzhou \\ 215006, China. Email: zhaojia0327@126.com; Dr. Xinliang Mao. Guangdong Key Laboratory of Protein Modification and Degradation, \\ School of Basic Medical Sciences, Guangzhou Medical University, 1 Xinzao Road, Guangzhou 511436, China. Email: xinliangmao@gzhmu.edu. \\ cn; Prof. Yufeng Xie. Department of Oncology, the First Affiliated Hospital of Soochow University, 188 Shizi Street, Suzhou 215006 , China. \\ Email: sdxyf@163.com.
}

Background: Cyclin D1 (CCND1) is overexpressed in non-small cell lung cancer (NSCLC) and contributes to its tumorigenesis and progression. Accumulating evidence shows that ubiquitin-specific protease 5 (USP5), an important member of the USP family, acts as a tumor promoter by deubiquitinating and stabilizing oncoproteins. However, neither the mechanism for dysregulated turnover of CCND1 protein nor the association of CCND1 with USP5 in NSCLC is well understood.

Methods: The association of USP5 with CCND1 in human NSCLC cells and clinical tissues was determined by immunoprecipitation/immunoblotting, immunohistochemistry (IHC), and The Cancer Genome Atlas database analyses. The effect of USP5 knockdown or overexpression on NSCLC cell proliferation in vitro was assessed by Cell Counting Kit-8, flow cytometry-based cell cycle, and colony formation assays. The effect of the USP5 inhibitor EOAI3402143 (G9) on NSCLC proliferation in vitro was analyzed by CCK-8 assay. The effect of G9 on NSCLC xenograft tumor growth was also examined in vivo, using athymic BALB/c nude mice.

Results: USP5 physically bound to CCND1 and decreased its polyubiquitination level, thereby stabilizing CCND1 protein. This USP5-CCND1 axis promoted NSCLC cell proliferation and colony formation. Further, knockdown of USP5 led to CCND1 degradation and cell cycle arrest in NSCLC cells. Importantly, this tumor-suppressive effect elicited by USP5 knockdown in NSCLC cells was validated in vitro and in vivo through chemical inhibition of USP5 activity using G9. Consistently, G9 downregulated the protein levels of CCND1 in NSCLC cells and xenograft tumor tissues. Also, the expression level of USP5 was positively associated with the protein level of CCND1 in human clinical NSCLC tissues.

Conclusions: This study has provided the first evidence that CCND1 is a novel substrate of USP5. The USP5-CCND1 axis could be a potential target for the treatment of NSCLC.

Keywords: Non-small cell lung cancer (NSCLC); ubiquitin-specific protease 5 (USP5); cyclin D1 (CCND1);

^ ORCID: 0000-0003-4848-2267. 
proliferation; ubiquitin-proteasome pathway

Submitted Jul 02, 2021. Accepted for publication Oct 22, 2021.

doi: $10.21037 /$ tlcr-21-767

View this article at: https://dx.doi.org/10.21037/tlcr-21-767

\section{Introduction}

Lung cancer is the most commonly diagnosed malignacy (11.6\% of total cases) and the leading cause of cancer-related deaths (18.4\% of total cancer deaths) $(1,2)$. According to its histopathological characteristics, lung cancer can be classified into small cell lung cancer and non-small cell lung cancer (NSCLC). Accounting for about $85 \%$ of all lung cancer cases (2-4), NSCLC is a large subtype of lung cancers that includes squamous cell carcinoma, adenocarcinoma, large cell carcinoma and other rare histologies. Despite the last decade witnessing advances in targeted therapy and immunotherapy for lung cancer, the 5-year survival rate of patients with the disease remains low (5). Therefore, gaining progressive understanding of the mechanisms of disease initiation and progression leads to the identification of biomarkers. The unveiling of potential therapeutic targets is instructive and meaningful to treating and improving the prognosis of NSCLC.

Cell cycle progression is determined by a well-tuned signal transduction cascade, in which D-type cyclins play a critical role. Human cyclin D genes encode three D-type cyclins, namely cyclin D1 (CCND1), cyclin D2 (CCND2), and cyclin D3 (CCND3), each of which can interact with and activate cyclin-dependent kinase 4/6 (CDK4/6). Once activated, CDK4/6 phosphorylates the retinoblastoma $(\mathrm{Rb})$ protein and promotes G1/S phase cell cycle transition $(6,7)$. Notably, CDK4/6 inhibitors have recently become the standard of care in several settings of breast cancer patients, pointing out the therapeutic relevance of this axis (8).

NSCLC has the characteristics of a high proliferation rate and rapid growth, which are highly associated with shorter cell cycle intervals and rapid cell mitosis. In NSCLC, CCND1 has been found to be elevated and to promote carcinogenesis, cell cycle progression, metastasis, and drug resistance (9-13). Furthermore, its overexpression is associated with a shorter overall survival (OS) rate in patients with lung cancer $(11,14)$. These reports suggest that CCND1 is a key driver marker and an important therapeutic target in NSCLC. Cyclin D1 is frequently dysregulated at multiple transcriptional and post-transcriptional levels in human cancers $(10,15)$. However, the regulatory mechanism of CCND1 expression in NSCLC, especially at a posttranslational level, has largely remained elusive.

CCND1 is a rapid response protein in cell cycle progression. It has a very short half-life, owing to the PEST domain at its C-terminus, which is characteristic of proteins that are rapidly turned over (11). Active glycogen synthase kinase- $3 \beta$ (GSK-3 $\beta$ ) can phosphorylate CCND1 protein at threonine residue 286 (T286), consequently triggering ubiquitin-mediated degradation in proteasome (16). It has also been reported that CCND1 can be polyubiquitinated by regulator of cullin 1 (ROC1)-cullin 1 (Cul1) (17) and F-box protein 4 (FBX4) (18), two major components of the Skp1-Cul1-F-box (SCF) family of E3 ubiquitin ligases for proteasomal degradation.

CCND1 ubiquitination is a dynamic process and certain deubiquitinases can remove the conjugated ubiquitin molecules. Some members of the ubiquitin-specific protease (USP) family, including USP2a (19), USP10 (20), and USP22 (21), have been reported to potentially deubiquitinate CCND1 protein and enhance its stability. Recent studies have demonstrated that CCND1 can be upregulated by USP5, another member of the USP family, in pancreatic cancer (22) and NSCLC (23); however, the mechanism of action is still unclear.

Given its deubiquitinating effect on multiple oncoproteins (24), the present study was performed to investigate whether USP5 upregulates the expression level of CCND1 protein in NSCLC via deubiquitination and stabilization of CCND1. A previous study has shown that inhibition of deubiquitinases using small-molecule inhibitors displays a tumor-suppressive activity (25). In order to provide a therapeutic strategy moving from our mechanistic insights, we further evaluated the potential anticancer activity of USP5 inhibition. We present the following article in accordance with the ARRIVE reporting checklist (available at https://dx.doi.org/10.21037/tlcr-21-767).

\section{Methods}

\section{Cell lines}

Human NSCLC cell lines such as A549 (KRAS.G12S), NCI-H1299 [H1299] (NRAS Q61K), NCI-H23 [H23] 
(KRAS G12C), NCI-H460 [H460] (KRAS Q61H), HCC827 (EGFR del 19), and NCI-H838 [H838] (KRAS WT), human bronchial epithelial cell line HBE, and human embryonic kidney cell line HEK293T which is an excellent tool cell line for transfection and immunoprecipitation (IP) experiments were purchased from Procell (Wuhan, Hubei, China). The NSCLC cell lines and HBE cell line were cultured in Roswell Park Memorial Institute 1640 (RPMI 1640, BasalMedia, Shanghai, China). The HEK293T cell line was cultured in Dulbecco's Modified Eagle's Medium (DMEM, BasalMedia). The mediums were supplemented with $10 \%$ fetal bovine serum (ExCell Bio, Shanghai, China) and 1\% penicillin/ streptomycin (Beyotime, Nantong, Jiangsu, China).

\section{Plasmids and small interfering RNAs}

Human USP5 expression plasmid with a Myc tag (MycUSP5), CCND1 expression plasmid with a Flag tag (FlagCCND1), and ubiquitin (Ub) expression plasmid with an HA tag (HA-Ub) were generated as described previously $(11,26)$. Small interfering RNAs (siRNAs) targeting human USP5 including USP5 siRNA (siUSP5) \#1 (5'-CCC AGC GAG UUG ACU ACA U-3') and siUSP5 \#2 (5'-GAU AGA CAU GAA CCA GCG GAU-3'), as well as scramble control (siNC) were obtained from Ribobio (Guangzhou, Guangdong, China).

\section{Antibodies and chemicals}

The following antibodies (Abs) were used: rabbit antiCCND1 [Cat. No. 2922 for IP/immunoblotting (IB) and 2978 for immunohistochemistry (IHC), CST, Danvers, MA, USA], rabbit anti-USP5 (Cat. No. 10473-1-AP, Proteintech, Wuhan, Hubei, China), rabbit anti-CCND2 (Cat. No. 2924, CST), rabbit anti-CCND3 (Cat. No. 26775-1-AP, Proteintech), mouse anti-CDK4 (Cat. No. 12790, CST), mouse anti-CDK6 (Cat. No. 3136, CST), mouse anti-Rb (Cat. No. 9309, CST), rabbit anti-PhosphoRb (Ser780) (Cat. No. 3590, CST), rabbit anti- $\beta$-tubulin (Cat. No. 10068-1-AP, Proteintech), mouse anti-Ub (Cat. No. sc-271289, Santa Cruz, Dallas, TX, USA), mouse antiMyc (Cat. No. M192-3, MBL, Tokyo, Japan), mouse antiFlag (Cat. No. M185-3, MBL), mouse anti-HA (Cat. No. M180-3, MBL), and horseradish peroxidase (HRP)-labeled goat anti-rabbit IgG $(\mathrm{H}+\mathrm{L})$ (Cat. No. A0208, Beyotime) and anti-mouse IgG $(\mathrm{H}+\mathrm{L})$ (Cat. No. A0216, Beyotime). Cycloheximide (CHX) was purchased from Sigma-Aldrich Chemicals (St. Louis, MO, USA). WP1130 (Degrasyn) and
G9 (EOAI3402143) were purchased from Selleck Chemicals (Houston, TX, USA).

\section{Transfection of plasmids and siRNAs}

For our experiments, plasmids and siRNAs were transfected into NSCLC or HEK293T cells, using Lipofectamine 2000 reagent (Invitrogen, Carlsbad, CA, USA), and the cells were collected at the post-transfection time points indicated.

\section{IB analysis}

Cells were lysed with RIPA lysis buffer (Beyotime) and the protein concentrations were measured by bicinchoninic acid (BCA) assay (Pierce, Rockford, IL, USA). Equal amounts of proteins $(30 \mu \mathrm{g})$ were subjected to sodium dodecyl sulfate-polyacrylamide gel electrophoresis and transferred to a polyvinylidene difluoride membrane (Merck Millipore, Billerica, MA, USA). Blots were probed with the appropriate Abs. Primary and secondary Abs were used at concentrations in line with the suppliers' instructions.

\section{IP assay}

The IP assay was performed as described previously (27). Briefly, Cell lysates were then prepared in a RIPA lysis buffer and centrifuged at $12,000 \mathrm{rpm}$ for $10 \mathrm{~min}$ at $4{ }^{\circ} \mathrm{C}$, the resultant proteins $(200 \mu \mathrm{g})$ were first incubated with each $\mathrm{Ab}$ of interest $(2 \mu \mathrm{g})$ for IP at $4{ }^{\circ} \mathrm{C}$ overnight and then with protein $\mathrm{A} / \mathrm{G}-\mathrm{Sepharose}$ beads (Beyotime) at $4{ }^{\circ} \mathrm{C}$ for 1 hour. The collected beads were then washed 3 times with cold PBS, and finally suspended in $20 \mu \mathrm{L}$ of $2 \times$ loading buffer and boiled for IB with the appropriate Abs.

\section{Cell cycle analysis}

After 48-hour knockdown of USP5, NSCLC cells were fixed with $70 \%$ pre-cooled ethanol for 24 hours. After being washed with cold phosphate-buffered saline (PBS), $2 \times 10^{6}$ cells from each group were stained with propidium iodide (Beyotime) for 30 minutes before flow cytometry was used to analyze the cell cycle profile.

\section{Cell proliferation and colony formation assays}

NSCLC cells were transfected with Myc-USP5 plasmid or empty vector plasmid (negative control) and specific siUSP5 or siNC (negative control) for 48 hours. For the 
cell proliferation assay, transfected cells were plated in 96well plates $\left(100 \mu \mathrm{L}\right.$ cell suspension; $5 \times 10^{3}$ cells per well) and cultured for 24,48 , and 72 hours. To examine cell viability, cells were incubated with $10 \mu \mathrm{L}$ of Cell Counting Kit8 (CCK-8) solution (Beyotime) for 1 hour before a plate reader was used to measure the optical density (OD) at $450 \mathrm{~nm}$. The relative cell viability was then analyzed. For the colony formation assay, transfected cells were plated into six-well plates at a density of 500 cells per well and cultured for two weeks. To determine the number of cell colonies, cells were subjected to fixing in $4 \%$ polyformaldehyde for 30 minutes, followed by staining with Giemsa solution for 20 minutes. In another in vitro cell proliferation assay, NSCLC cells were treated with WP1130 (28) or G9 (29), a USP5 inhibitor, at various final concentrations $(0,2,4,6$, and $8 \mu \mathrm{M}$ ) for 24,48 , and 72 hours, and then subjected to CCK-8 assay as described above.

\section{Mouse xenograft study}

Male BALB/c nude mice (4-5 weeks old, 16-18 g; Shanghai SLAC Laboratory Animal Co., Ltd., Shanghai, China) were injected subcutaneously with $1 \times 10^{7}$ A549 NSCLC cells suspended in $0.2 \mathrm{~mL}$ of Matrigel (BD, San Diego, CA, USA) and RPMI-1640 mixture (Volume Matrigel: $_{\text {Volume }}$ RPMI-1640 $=1: 1$ ). After the tumors had grown to about $100 \mathrm{~mm}^{3}$, the mice were randomly allocated into two groups (six mice per group) and then subjected to treatment. One group was intraperitoneally injected with G9 at a dose of $15 \mathrm{mg} / \mathrm{kg}$ body weight in dimethyl sulfoxide:polyethylene glycol 300:Tween-80: $\mathrm{ddH}_{2} \mathrm{O}$ for 24 days (at 10-11 A.M.), and the other group was given vehicle only (negative control). The body weights and tumor volumes of the mice were monitored at designated intervals. At the experiment's end, the tumor-bearing mice were humanely euthanized by continuous inhalation with $30 \% \mathrm{CO}^{2}$ for 5 minutes. Mice with the absence of a heartbeat for 30 seconds accompanied by no toe-pinch reflex were considered to be dead. The xenograft tumors were then collected for further evaluation. The mice were maintained in a specific pathogenfree animal facility (dark/light cycle: 12 hours/12 hours) at Soochow University (Suzhou, Jiangsu, China).

\section{Tissue specimens}

Paraffin-embedded tissue specimens including lung adenocarcinoma (LUAD) tissues and normal lung tissues were collected from individuals treated in the Department of Pathology at the First Affiliated Hospital of Soochow
University between January 1, 2020 and August 30, 2020.

\section{IHC assay}

All tissue section samples were first deparaffinized with xylene and rehydrated with ethanol. Following antigen retrieval, the sections were incubated with $10 \%$ normal goat serum at $37{ }^{\circ} \mathrm{C}$ for 15 minutes. The sections were incubated with anti-USP5 (1:100) or anti-CCND1 (Cat. No. 2978 for IHC; 1:50) primary Ab at $4{ }^{\circ} \mathrm{C}$ overnight, and then with HRP-conjugated goat anti-rabbit $\operatorname{IgG}(1: 200)$ secondary Ab. Finally, the sections were reacted with diaminobenzidine and counterstained with hematoxylin. The IHC-stained sections were assessed under a microscope by two histopathologists, and the semi-quantitative IHC scores of USP5 and CCND1 were recorded, ranging from 0 (minimum score) to 12 (maximum score) (30).

\section{Ethical statement}

All animal experiments were reviewed and approved by the Review Board of Animal Care and Use of Soochow University (Institutional Review Board No. 202008A619), and were performed in compliance with its institutional guidelines for the care and use of laboratory animals. The human study has been conducted in accordance with the Declaration of Helsinki and approved guidelines. The research using human specimens was approved by the Ethics Committee of the First Affiliated Hospital of Soochow University (Institutional Review Board no. 2021036). Informed consent was taken from all the patients.

\section{Statistical analysis}

Comparisons between the control and experimental groups were analyzed using Student's $t$-test, Kruskal-Wallis test, or Log-rank test. SPSS20.0 (SPSS, Chicago, IL, USA) was employed for all statistical analyses. A $P$ value of less than 0.05 ( $^{*}, \mathrm{P}<0.05,{ }^{* *}, \mathrm{P}<0.01$, and $\left.{ }^{* * *}, \mathrm{P}<0.001\right)$ was considered significant.

\section{Results}

\section{USP5 stabilizes and upregulates CCND1 protein in NSCLC cells}

A previous report (22) on short hairpin RNA (shRNA) barcode library screening showed that USP5 can upregulate the level 
of CCND1 protein in pancreatic cancer cells. Studies have also shown that USP5 can powerfully deubiquitylate and stabilize some proteins, such as FoxM1 STAT3 and c-Maf (24). To determine whether USP5 can elevate CCND1 in such a modulatory way, we first examined the effect of USP5 on CCND1 by co-transfecting the tool cell line HEK293T with Flag-CCND1 and Myc-USP5 plasmids. We found that USP5 increased the protein level of CCND1 in a concentrationdependent (Figure 1A) and time-dependent (Figure 1B) manner. We next examined the half-life of CCND1 protein in the presence of USP5 and CHX, a specific inhibitor of protein synthesis de novo. The CHX chase assay showed that USP5 markedly prolonged the half-life of $C C N D 1$ protein $(\mathrm{P}<0.001)$ (Figure 1C,1D), extending it from less than 30 minutes to more than 4 hours. To further examine whether this regulatory effect of USP5 on CCND1 could also occur in NSCLC cells, we analyzed the effect of USP5 overexpression or knockdown on the protein levels of endogenous CCND1 in the A549 and H1299 NSCLC cell lines. Interestingly, USP5 overexpression remarkably increased the levels of CCND1 protein in NSCLC cells (Figure 1E), whereas knockdown of USP5 using its specific siRNA reduced the protein levels of CCND1 (Figure 1F). We further evaluated the association of the endogenous expression of USP5 and CCND1 in a panel of NSCLC cell lines. As shown in Figure $1 G, 1 H$, the protein level of CCND1 was positively correlated with USP5 expression in NSCLC cells $(\mathrm{P}<0.01)$. Taken together, the above results indicate that USP5 is capable of upregulating CCND1 in NSCLC cells to a great extent by increasing its protein stability.

\section{USP5 interacts with CCND1 and decreases its polyubiquitination}

Given that USP5 is a deubiquitinating enzyme (24), we wondered whether USP5 could directly interact with and deubiquitinate CCND1 to stabilize CCND1 protein. To this end, we subjected HEK293T cells to cotransfection with Myc-USP5 and Flag-CCND1 plasmids followed by an IP/ IB assay. As shown in Figure 2A, CCND1 (Flag) was present in the anti-Myc (for Myc-USP5 IP) immunoprecipitates. In the reciprocal IP/IB assay, USP5 (Myc) was also confirmed to be present in the anti-Flag (for Flag-CCND1 IP) immunoprecipitates (Figure $2 B$ ). Importantly, the experiment could be recapitulated in NSCLC cells. As shown in Figure $2 C$, endogenous CCND1 was detected in the anti-Myc (for Myc-USP5 IP) immunoprecipitates from both A549 and H1299 cell lysates, evidencing that these two proteins, USP5 and CCND1, could directly bind with each other in NSCLC cells. Next, the CCND1 polyubiquitination level was measured in the presence of USP5. As shown in Figure 2D, ectopic expression of USP5 markedly reduced the polyubiquitination level of CCND1 in HEK293T cells. Further, our gain- and loss-of-function experiments demonstrated that overexpression of USP5 prevented endogenous CCND1 from polyubiquitinating in NSCLC cells and increased the CCND1 protein level (Figure 2E), whereas knockdown of USP5 produced the opposite effect (Figure 2F). Collectively, these findings indicate that USP5 stabilizes CCND1 by directly binding to CCND1 and decreasing its polyubiquitination level.

\section{Knockdown of USP5 impedes cell cycle transition in NSCLC cells}

Since CCND1, as a member of the D-type cyclin subfamily, is vital for cell cycle progression (9-11), we hypothesized that the USP5-elicited regulatory effect on CCND1 expression would affect cell cycle transition in NSCLC cells. To this end, flow cytometry was used to analyze NSCLC cell cycle distribution following USP5 knockdown. As shown in Figure $3 A, 3 B$, knockdown of USP5 increased the proportions of A549 and H1299 cells in the G1 phase $(\mathrm{P}<0.001$ or $<0.05)$. Through its associations with CDK4 and CDK6, CCND1 has been shown to induce $\mathrm{Rb}$ phosphorylation $(6,7,9)$, thereby promoting cell cycle progression. Therefore, we next studied the effects of USP5 on CCND1-CDK4/6-Rb signaling in NSCLC cells. Our data revealed that USP5 knockdown did not change the expression level of CDK4 or CDK6 but suppressed the phosphorylation level of $\mathrm{Rb}$ (Ser780), and was accompanied by the downregulation of CCND1 (Figure 3C). We also assessed the effects of USP5 on other close members of the D-type cyclin subfamily, such as CCND2 and CCND3. Our data showed that knockdown of USP5 decreased the level of CCND1 but not that of CCND2 or CCND3 in NSCLC cells (Figure 3C), which suggested that USP5-mediated deubiquitylation and stabilization of D-type cyclins is specific to CCND1. Collectively, these results showed that USP5 indeed reprograms NSCLC cell cycle progression, with its knockdown arresting cell cycle transition.

\section{USP5 promotes NSCLC cell proliferation and colony formation}

Overexpression of CCND1 results in rapid cell growth owing to the bypass of the key cell-cycle checkpoints (6-11). To uncover whether USP5 can alter NSCLC cell growth by 


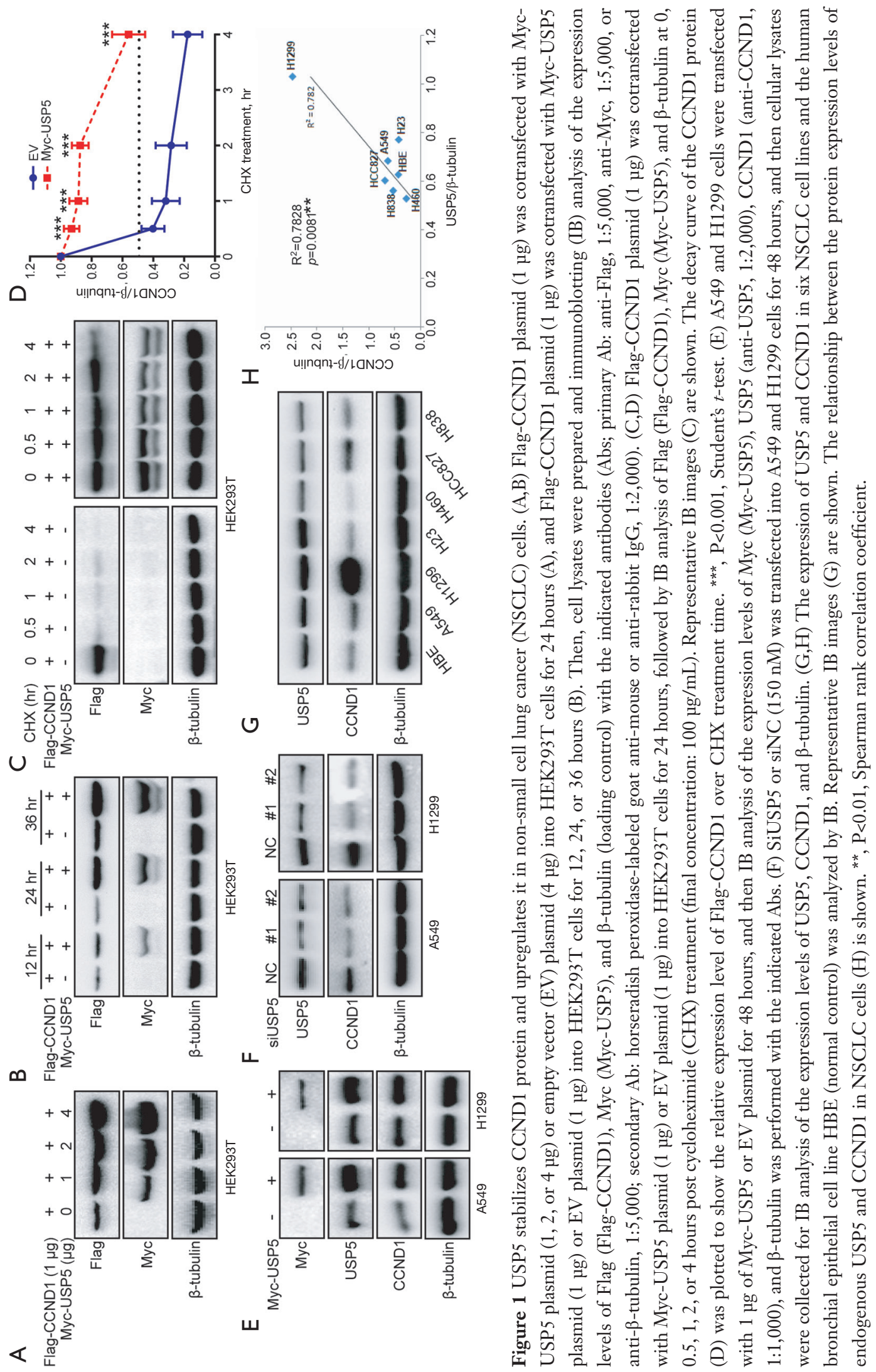



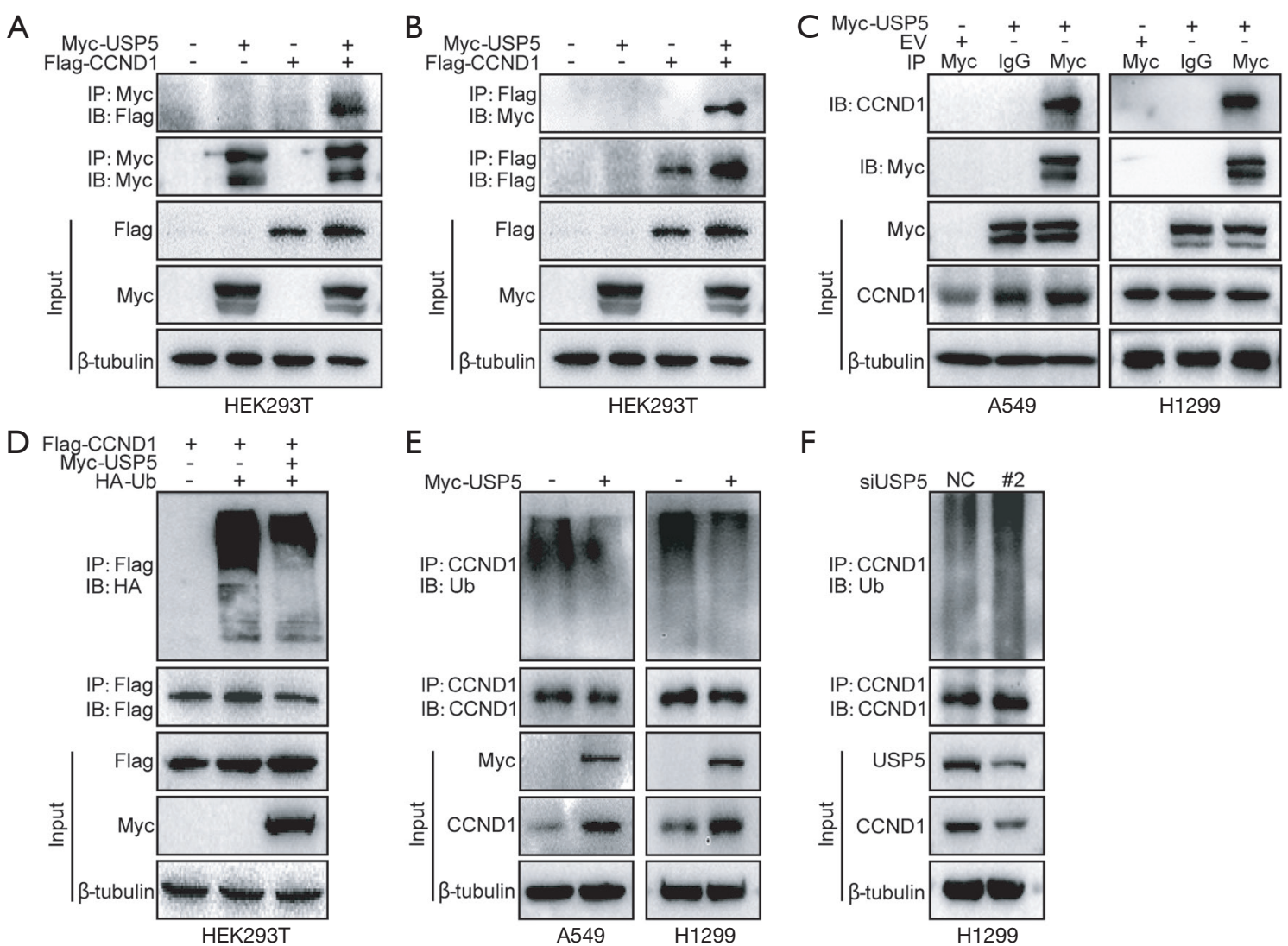

$\mathrm{F}$

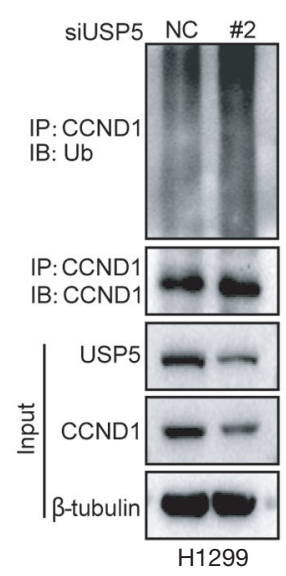

Figure 2 USP5 interacts with CCND1 and downregulates its polyubiquitination level. (A,B) Myc-USP5 or EV plasmid (1 $\mu$ g) was cotransfected with Flag-CCND1 or empty vector (EV) plasmid $(1 \mu \mathrm{g})$ into HEK293T cells. After 24 hours of transfection, cells were collected for lysate preparation. Equal amounts of proteins $(200 \mu \mathrm{g})$ were first subjected to immunoprecipitation (IP) with $2 \mu \mathrm{g}$ of anti-Myc (A) or anti-Flag (B) antibody (Ab), and subsequent immunoblotting (IB) with the indicated Abs. (C) Direct protein-protein interaction between Myc-USP5 and endogenous CCND1 was assessed in the non-small cell lung cancer (NSCLC) cell lines A549 and H1299. Myc-USP5 or EV plasmid $(1 \mathrm{\mu g})$ was transfected into A549 and H1299 cells for 48 hours. Cells were collected for lysate preparation. The cell lysates were then subjected to IP with anti-Myc or normal rabbit IgG (negative control) Ab and subsequent IB with the indicated Abs. (D) Flag-CCND1 plasmid (1 $\mu \mathrm{g}$ ) was cotransfected with Myc-USP5 and HA-Ub plasmids (1 $\mu \mathrm{g} / \mathrm{each})$, with EV and HA-Ub plasmids (1 $\mu \mathrm{g} / \mathrm{each})$, or with EV plasmids (1 $\mu \mathrm{g} / \mathrm{each})$ into HEK293 $\mathrm{T}$ cells for 24 hours. Cell lysates were then prepared for IP with anti-Flag Ab and subsequent IB analysis of HA (anti-HA, 1:5,000) and Flag with indicated Abs. (E) NSCLC cell lines A549 and H1299 were transfected with Myc-USP5 or EV plasmid $(1 \mu \mathrm{g})$ for 48 hours. Cell lysates were prepared for IP with anti-CCND1 Ab followed by IB analysis of Ub (anti-Ub, 1:1,000) and CCND1 with indicated Abs. (F) SiUSP5 or siNC $(150 \mathrm{nM})$ was transfected into H1299 cells. After 48 hours, cell samples were prepared for IP/IB as indicated above. IB analysis of input served as a positive control for all IP/IB assays.

stabilizing CCND1, the Myc-USP5 plasmid and its specific siRNA were transfected into A549 and H1299 NSCLC cells and cell proliferation assays were subsequently performed. As shown by the CCK-8 assay, A549 and H1299 cell proliferation was promoted by the overexpression of USP5 but impaired by its knockdown (Figure 3D,3E). The colony formation assay further revealed that knockdown of USP5 also markedly reduced the number of colonies formed, both by A549 and H1299 cells (Figure 3F,3G). These findings are consistent with the effects of USP5 on CCND1 expression and cell cycle arrest in NSCLC cells.

\section{Inbibition of USP5 activity suppresses NSCLC cell proliferation in vitro and tumor growth in vivo}

The above results showed that USP5 could promote NSCLC 
A
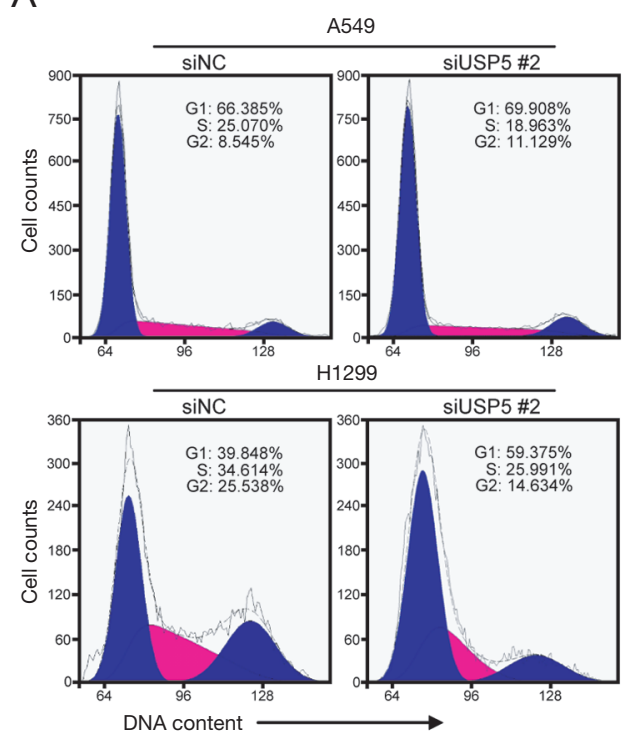

D

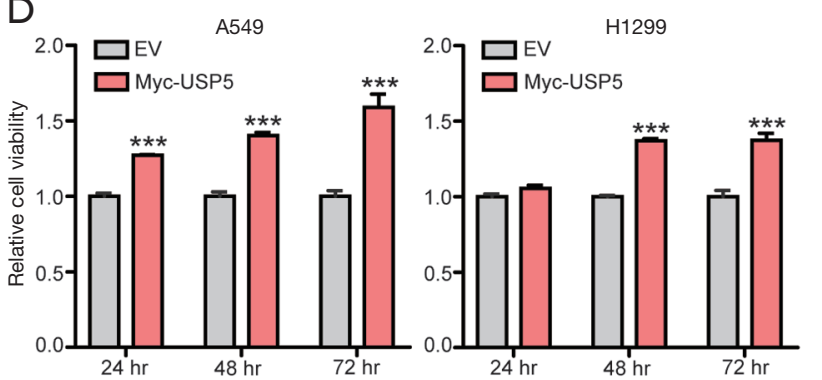

$\mathrm{F}$
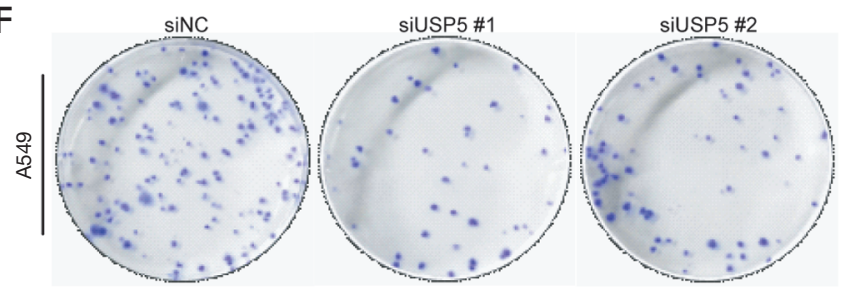

B
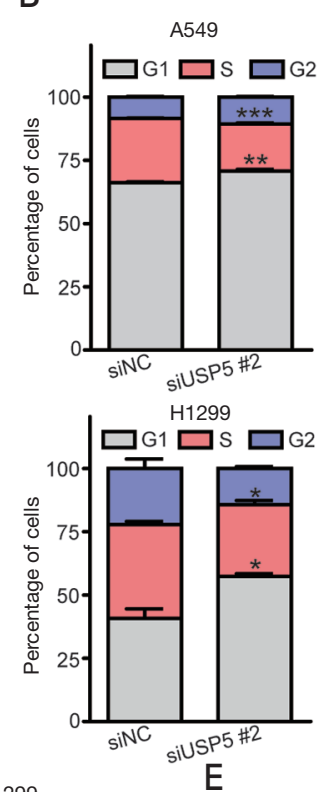

$\mathrm{E}$
C

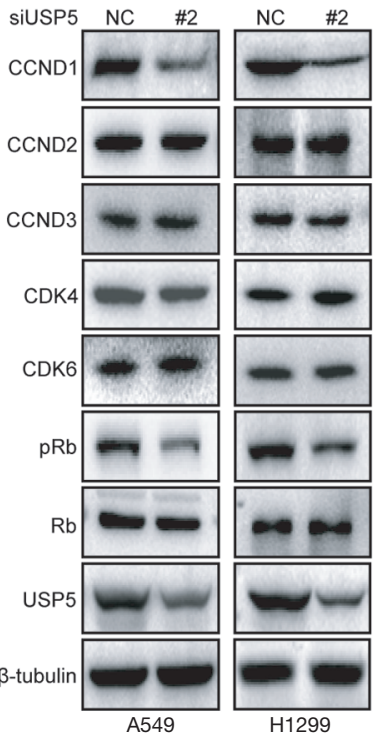

G

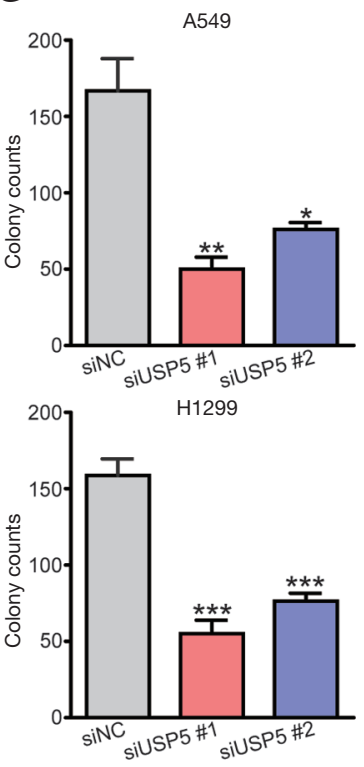

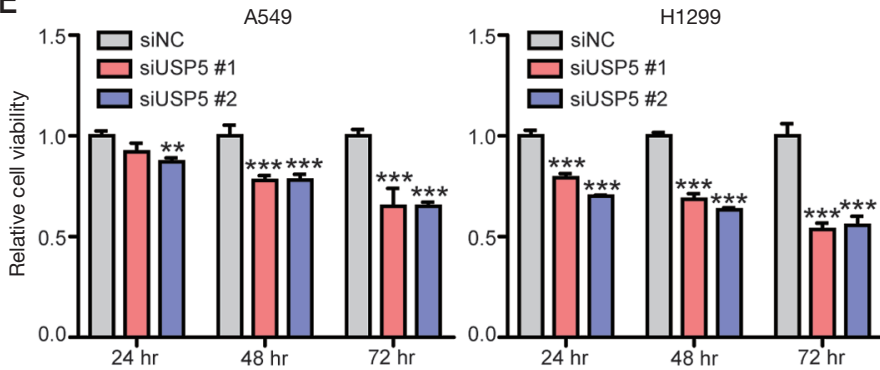
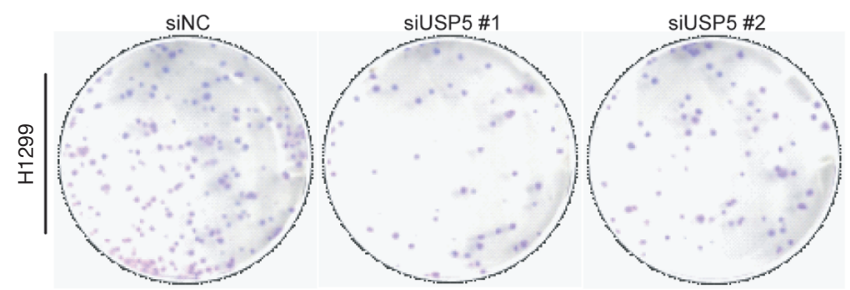

Figure 3 USP5 promotes cell cycle progression and proliferation in non-small cell lung cancer (NSCLC) cells. (A,B) A549 and H1299 cells were transfected with siUSP5 or siNC $(150 \mathrm{nM})$ for 48 hours. Cell cycle phases were then analyzed by flow cytometry. Representative images of flow cytometric cell cycle analysis (A) and the percentage of cells in each cell cycle phase distribution (B) are shown. G1 or S in A549: G1, ${ }^{* *}, \mathrm{P}<0.01$ or $\mathrm{S},{ }^{* * *}, \mathrm{P}<0.001$; $\mathrm{G} 1$ or $\mathrm{S}$ in H1299: ${ }^{*} \mathrm{P}<0.05$, Student's $t$-test. (C) In A549 and H1299 cells, the levels of proteins involved in the CCND1-CDK4-Rb signaling pathway after knockdown of USP5 were assessed by immunoblotting (IB) assay using the indicated antibodies (Abs) [primary Ab: anti-CCND1, 1:1,000, anti-CCND2, 1:1,000, anti-CCND3, 1:1,000, anti-CDK4, 1:1,000, antiCDK6, 1:1,000, anti-pRb (Ser780), 1:1,000, or anti-Rb, 1:1,000; secondary Ab: horseradish peroxidase-labeled goat anti-rabbit or antimouse IgG, 1:2,000]. (D,E) Myc-USP5- and siUSP5-transfected A549 and H1299 cells along with the respective control cells were cultured for different time periods (24-72 hours), and cell viability was then measured by Cell Counting Kit-8 assay. ${ }^{* *}, \mathrm{P}<0.01$ or ${ }^{* * *}, \mathrm{P}<0.001$ compared with their respective control at the same time point, Student's $t$-test. (F,G) Colony formation assay was performed using siUSP5tranfected A549 and H1299 cells and their respective control cells. Representative images of colony formation assay (F) and the number of colonies (G) are shown. * $\mathrm{P}<0.05$, **, $\mathrm{P}<0.01$, or ${ }^{* * *}, \mathrm{P}<0.001$ compared with the respective control, Student's $t$-test. 
cell proliferation via upregulating the CCND1 protein level through deubiquitylation and stabilization of CCND1. Therefore, we wondered whether USP5 could serve as an effective therapeutic target for NSCLC that could be pharmacologically targeted. To explore this idea, the USP5 chemical inhibitors WP1130 (28) and G9 (29) (Figure 4A) were utilized as therapeutic approaches to treating NSCLC. As shown in Figure 4B, the effects of WP1130 and G9 in suppressing the proliferation and viability of A549 and H1299 NSCLC cells in vitro were closely comparable. In line with the above finding that genetic inhibition of USP5 suppressed the level of CCND1 protein, chemical inhibition of USP5 activity using WP1130 and G9 also produced a similar effect of downregulating the protein level of CCND1 in NSCLC cells (Figure 4C). As expected, neither WP1130 nor G9 affected the expression level of USP5 (Figure 4C).

We next explored whether chemical suppression of USP5 activity could inhibit NSCLC tumor growth in mice in vivo. To this end, a human NSCLC xenograft model was established by subcutaneously injecting athymic nude mice with A549 NSCLC cells and then subjecting them to treatment with G9, which has better solubility and inhibitory activity against USP5 than does WP1130 (29). The growth of the A549 NSCLC xenograft tumors was monitored before and after treatment. At the end of the animal study, all mice were healthy and included for analysis ( $\mathrm{n}=6$ in each group). The tumors in the G9-treated group were significantly smaller than those in the vehicletreated group (Figure 4D-4F). The tumor weight also exhibited a striking decrease in the G9-treated group in comparison with the vehicle group $(\mathrm{P}<0.001)$ (Figure $4 G)$. Most importantly, the body weight of the G9 group was slightly decreased compared to that of the vehicle group (Figure $4 H$ ), which is consistent with a previous report (29). Biological analyses further demonstrated that G9 did not alter the levels of alanine aminotransferase (Figure 4I), aspartate aminotransferase (Figure 4f), creatinine (Figure 4K), or urea (Figure 4L) in the blood of the mice, indicating that treatment with $15 \mathrm{mg} / \mathrm{kg}$ of G9 was well tolerated. Our data showed that the inhibition of USP5 activity with G9 also effectively suppressed NSCLC tumor growth in vivo, with no significant toxicity. To further evaluate the in vivo effect of G9 on the expression levels of CCND1 and USP5 in the mouse tumors, all tumor tissues were subjected to IHC assay and each was given a staining score. As shown in Figure $4 M, 4 N$, the expression levels of CCND1 protein were significantly reduced in the G9treated group $(\mathrm{P}<0.001)$, whereas the expression levels of USP5 protein were virtually unaffected, suggesting that G9 did decrease expression level of CCND1 protein in vivo by suppressing USP5 activity. These results indicated that the USP5-CCND1 axis could potentially be targeted in NSCLC via pharmacological inhibition of USP5 activity.

\section{USP5 expression level shows a positive association with the protein level of CCND1 in LUAD clinical tissues}

To assess the expression level of USP5 in human NSCLC clinical tissues, we first analyzed the data on USP5 mRNA expression in 535 LUAD tissues and 59 normal lung tissues from The Cancer Genome Atlas (TCGA) database. As shown in Figure $5 A$, the levels of USP5 mRNA were elevated in LUAD tissues $(\mathrm{P}<0.001)$. IHC staining of 76 LUAD tissues and 87 normal lung tissues was performed, and LUAD patients included 34 males and 42 females with an average age of 48.6 years. Our IHC analysis showed that the levels of USP5 protein were also upregulated in LUAD tissues $(\mathrm{P}<0.001)$ (Figure 5B,5C), which was consistent with a previous report (23). Additionally, TCGA data (Figure $5 D$ ) indicated that the expression levels of USP5 in stage IV LUAD tissues were higher than those in stage I tissues $(\mathrm{P}<0.01)$. To validate our NSCLC cell model data on the association of USP5 with CCND1, we further extracted the TCGA data on the CCND1 mRNA expression profile and performed an IHC analysis of CCND1 protein expression in LUAD and normal lung tissues using the same specimens as above. Although no significant difference was found in the mRNA expression levels of CCND1 between LUAD and normal lung tissues (Figure 5E), the CCND1 protein levels in the LUAD tissues were significantly higher than those in the normal lung tissues (Figure 5F,5G). Moreover, clinical data confirmed that the expression level of USP5 was positively correlated with the protein level of CCND1 in LUAD tissues $(\mathrm{P}<0.01)$ (Figure $5 H)$. The above results also highlight that the USP5-elicited increase in the stability of CCND1 protein is an important mechanism of the upregulation of USP5 in NSCLC.

\section{Correlation of the expression levels of USP5 and CCND1 with the prognosis of LUAD patients}

To further evaluate the prognosis significance of USP5 and CCND1 in LUAD, we analyzed the relationship of the mRNA and protein expression levels of USP5 and CCND1 with the OS of LUAD patients via TCGA (UALCAN) and The Human Protein Atlas (THPA) databases, respectively. UALCAN database (Figure $6 \mathrm{~A}$ ) showed that the mRNA level of USP5 was inversely 
A<smiles>CCC[C@H](NC(=O)/C(C#N)=C/c1cccc(Br)n1)c1ccccc1</smiles><smiles>CCCC(NC(=O)/C(C#N)=C/c1nc(Cl)ccc1Cl)c1ccc(OC)cc1</smiles>

G9

D

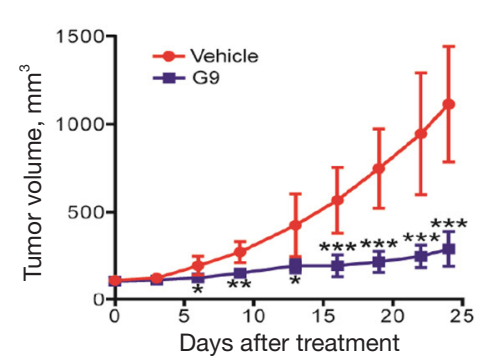

$\mathrm{H}$

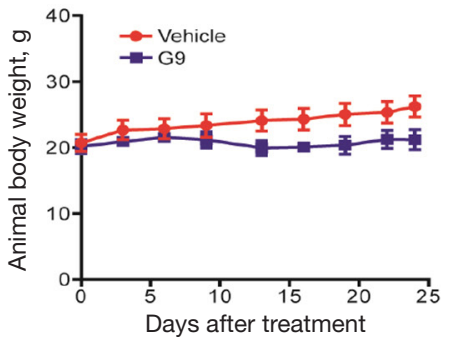

$\mathrm{L}$

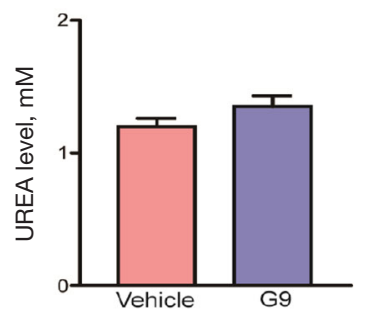

M
B

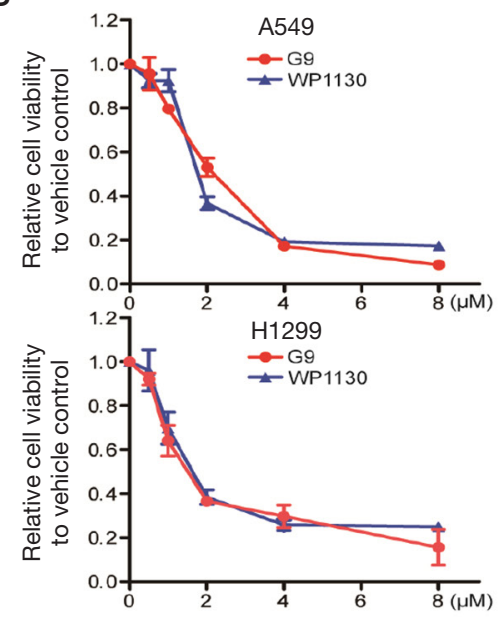

E

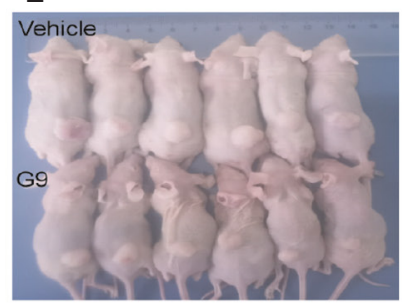

$\mathrm{F}$
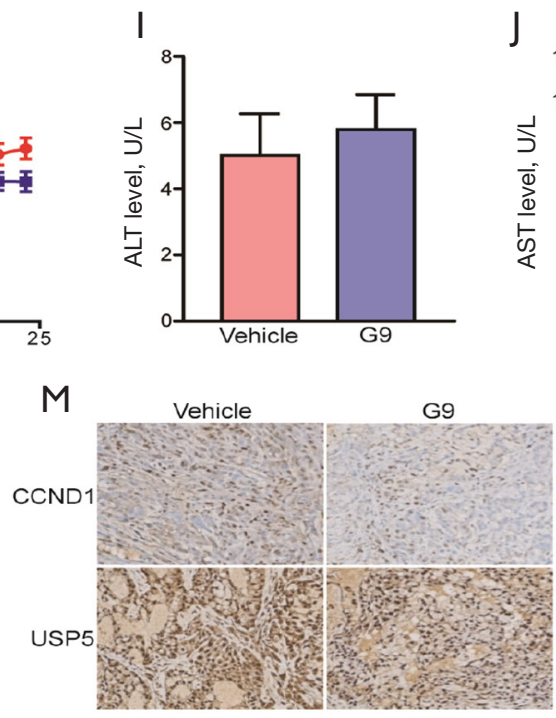

$\mathrm{N}$
C

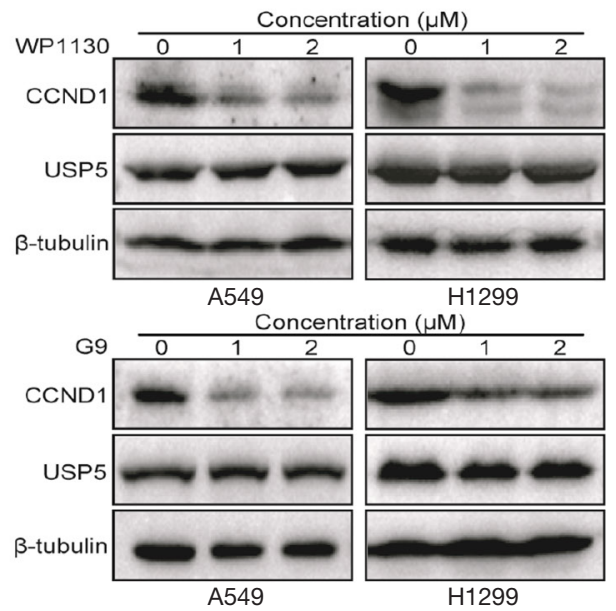

G

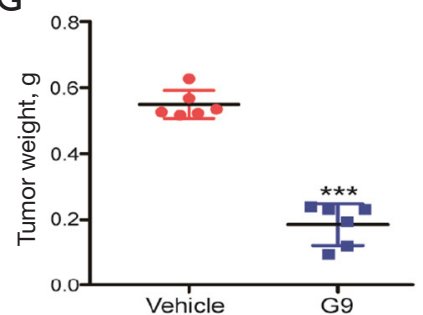

K
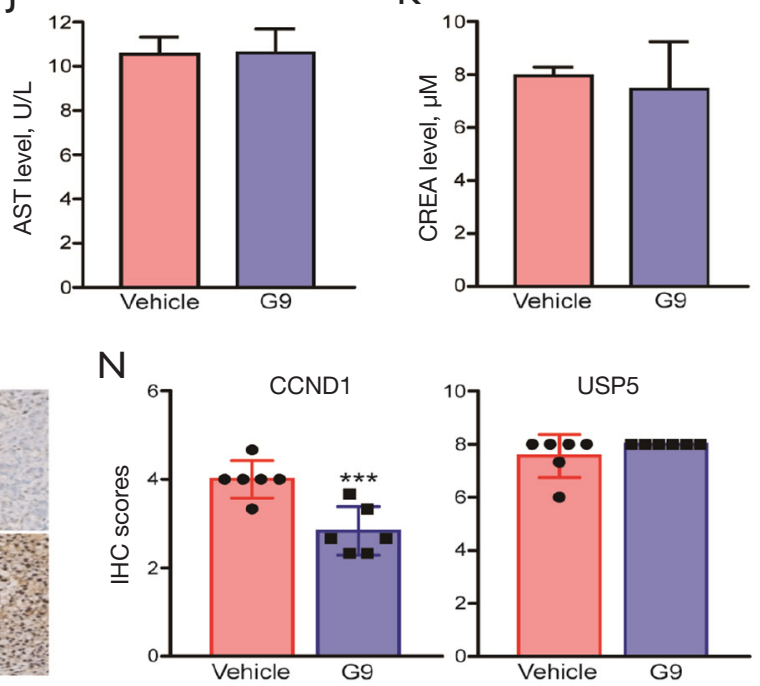

Figure 4 Inhibition of USP5 activity suppresses non-small cell lung cancer (NSCLC) cell proliferation and xenograft tumor growth. (A) The chemical structure of the USP5 inhibitors, WP1130 and EOAI3402143 (G9). (B) Both WP1130 and G9 inhibited the cell viability of A549 and H1299 cells in a concentration-dependent manner in vitro. Representative data under 48 hours of treatment are shown. (C) The effects of WP1130 and G9 on the expression levels of CCND1 and USP5 in NSCLC cells. A549 and H1299 cells were subjected to WP1130 or G9 treatment for 24 hours, followed by immunoblotting assay as indicated above. (D-G) G9 suppresses NSCLC tumor growth in nude mice. Tumor volume (D), images of tumor-bearing mice (E) and A549 xenograft tumors (F), and tumor weight (G) are shown. (H-L) G9 $(15 \mathrm{mg} / \mathrm{kg}$ ) exerted no significant toxicity in nude mice. The body weight of the mice $(\mathrm{H})$ and biological analyses of the levels of alanine aminotransferase (I), aspartate aminotransferase $(\mathrm{J})$, creatinine $(\mathrm{K})$, and urea $(\mathrm{L})$ in the blood of the mice are shown. (M,N) Immunohistochemical (IHC) analysis of the protein levels of CCND1 and USP5 in tumor tissues. Representative IHC images (upper: $\times 40$; lower: $\times 200)(\mathrm{M})$ and scores $(\mathrm{N})$ are shown. ${ }^{*}, \mathrm{P}<0.05 ;{ }^{* *}, \mathrm{P}<0.01$; ${ }^{* * *}, \mathrm{P}<0.001$, Student's $t$-test. 
A

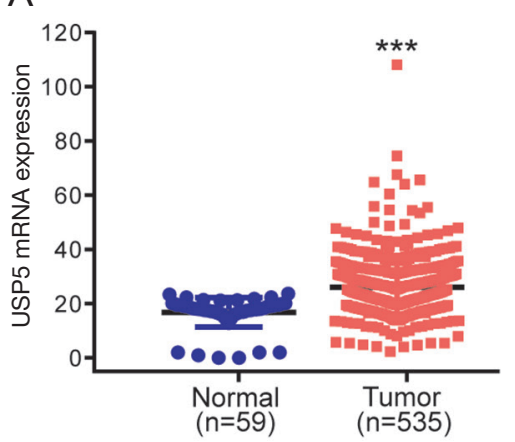

C

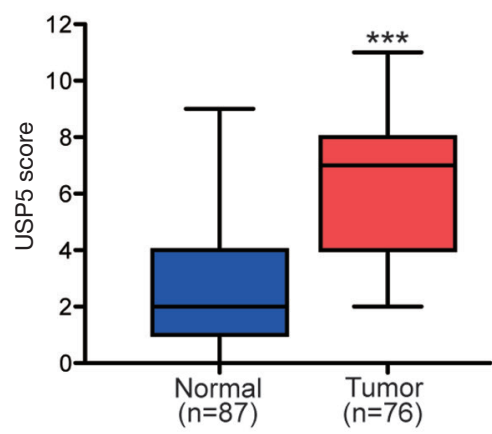

D

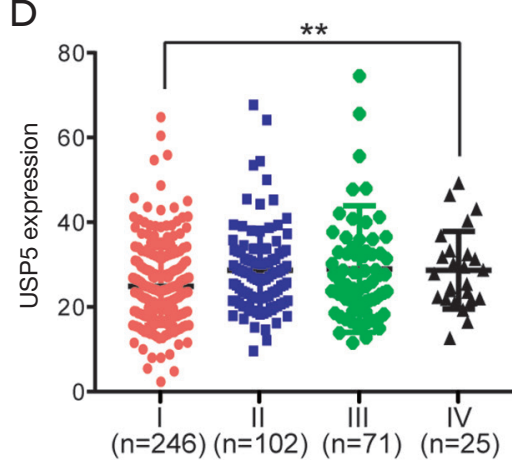

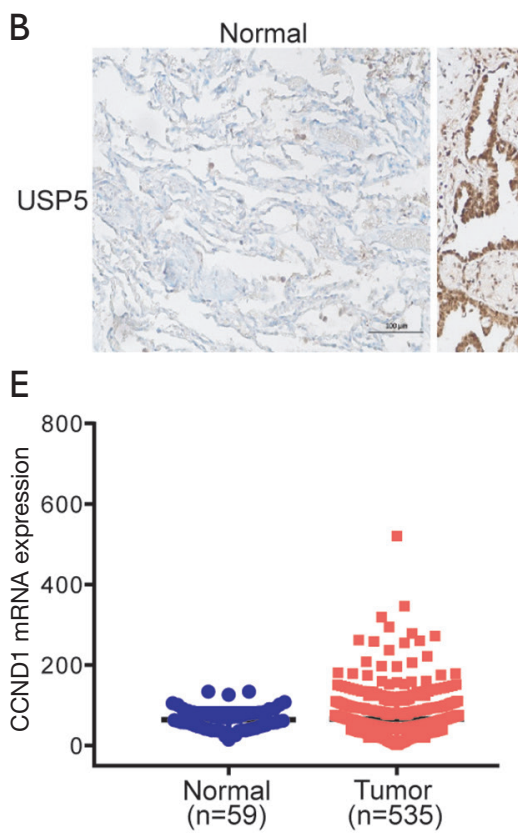

Tumor

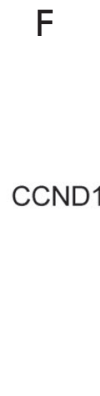

G

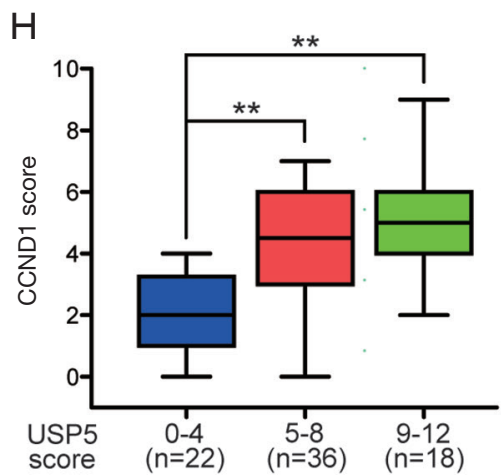

Figure 5 USP5 expression is positively associated with the expression of CCND1 at the protein but not at the mRNA level. (A) Expression levels of USP5 mRNA in lung adenocarcinoma (LUAD) tissues ( $\mathrm{n}=535$ ) and normal lung tissues ( $\mathrm{n}=59$ ) cited from The Cancer Genome Atlas (TGCA) dataset (http://cancergenome.nih.gov/). *** $\mathrm{P}<0.001$, Student's $t$-test. (B,C) Immunohistochemical (IHC) analysis of the protein levels of USP5 in clinical LUAD tissues $(n=76)$ and normal lung tissues $(n=87)$. Representative images $(\times 200)(B)$ and scores (C) of IHC analysis of USP5 are shown. ${ }^{* * *}, \mathrm{P}<0.001$, Student's $t$-test. (D) The association of USP5 level with tumor node metastasis (TNM) staging cited in the TCGA dataset. ${ }^{* *}, \mathrm{P}<0.01$, Student's $t$-test. (E) Expression levels of CCND1 mRNA in LUAD tissues (n=535) and normal lung tissues $(n=59)$ cited from the TCGA dataset. (F,G) IHC analysis of the protein levels of CCND1 in clinical LUAD tissues $(n=76)$ and normal lung tissues $(n=87)$. Representative images $(\times 200)(F)$ and scores (G) of IHC analysis of CCND1 are shown. ${ }^{* * *}, \mathrm{P}<0.001$, Student's $t$-test. $(\mathrm{H})$ The clinical association of USP5 protein level with CCND1 protein level in LUAD tissues. **, $\mathrm{P}<0.01$, Kruskal-Wallis test.

associated with the OS of LUAD patients $(\mathrm{P}<0.05)$. THPA database (Figure $6 B$ ) also showed that higher level of USP5 protein was related with shorter OS $(\mathrm{P}<0.001)$. In addition, although the mRNA level of CCND1 exhibited no correlation with LUAD patients' OS (Figure 6C), the protein level of CCND1 displayed a negative association with it $(\mathrm{P}<0.05)$ (Figure 6D). These data demonstrates that the expression levels of USP5 (either mRNA or protein) and CCND1 (only protein) are positively correlated with poor prognosis of LUAD patients, suggesting that USP5CCND1 axis promotes the malignant progression and leads to poor prognosis in NSCLC. 

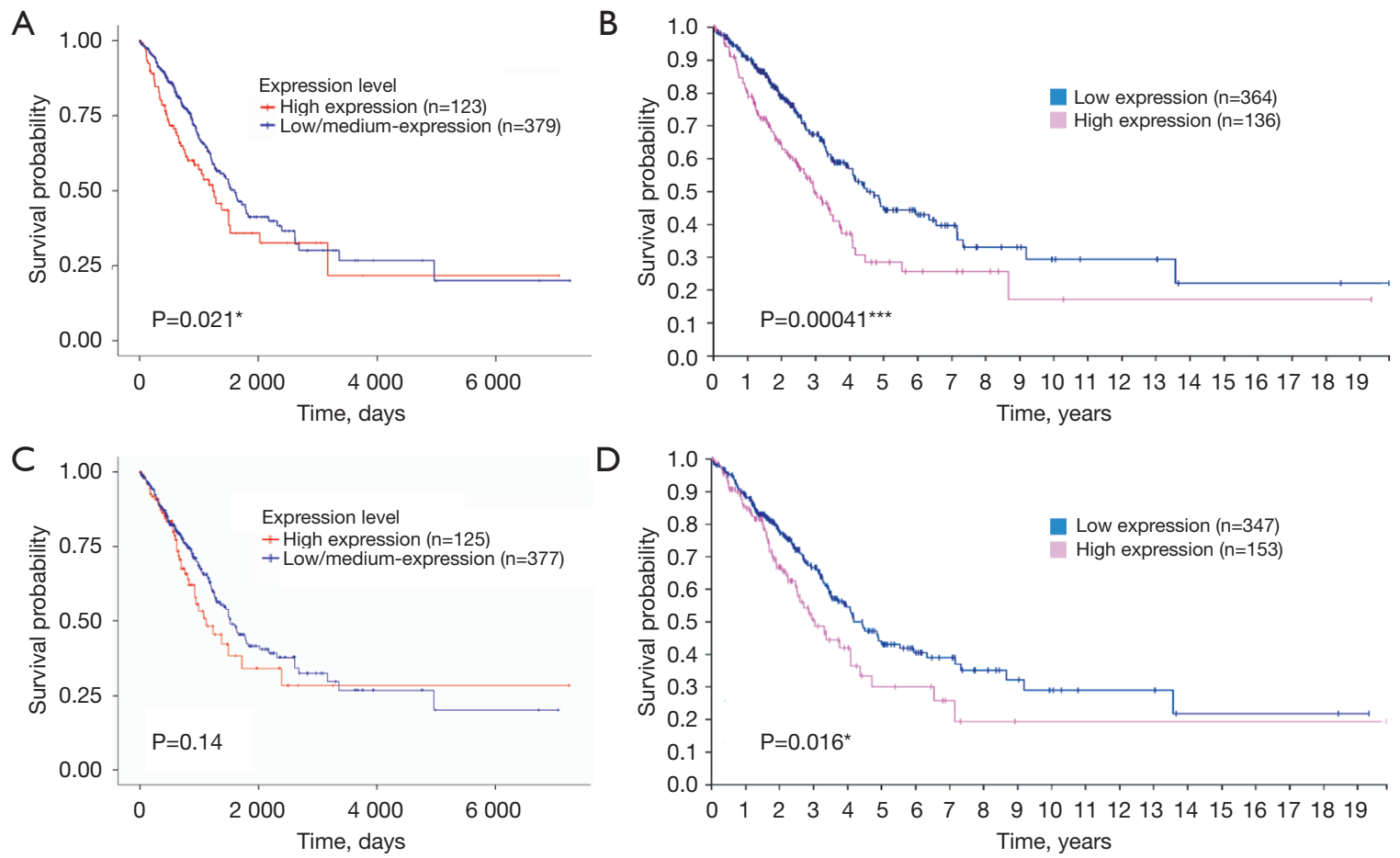

Figure 6 The expression level of USP5 and CCND1 is a negative index for patient survival in lung adenocarcinoma (LUAD) tissues. (A) Correlation of the mRNA level of USP5 with the prognosis of LUAD patients. * $\mathrm{P}<0.05$, Log-rank test. The original data were obtained from UALCAN database (http://ualcan.path.uab.edu/). (B) Correlation of the protein level of USP5 with the prognosis of LUAD patients. ***, $\mathrm{P}<0.001$, Log-rank test. The original data were obtained from The Human Protein Atlas (THPA) database (https://www.proteinatlas. org/). (C) Correlation of the mRNA level of CCND1 with the prognosis of LUAD patients. The original data were obtained from UALCAN database. (D) Correlation of the protein level of CCND1 with the prognosis of LUAD patients. *, P<0.05, Log-rank test. The original data were obtained from THPA database. All patients were classified into two groups based on the mRNA or protein expression level of USP5 or CCND1.

\section{Discussion}

Lung cancer is the leading cause of cancer-related death worldwide (1). The prognosis for patients with late or advanced NSCLC is extremely poor due to the absence of effective treatment modalities. NSCLC is a heterogeneous disease, the initiation and progression of which mainly result from the overexpression/overactivation or mutation/ loss of function of multiple genes. However, the details of the effects and mechanisms of these alteration genes, especially the protein ubiquitination-associated genes, are largely unknown. Given the broad functions of ubiquitination modification in various biological events and disorders including cancer $(31,32)$, identifying the vital ubiquitinases or deubiquitinases involved in lung cancer progression and exploring their potential as therapeutic targets are essential. In this context, we demonstrated that the deubiquitinase USP5 is capable of directly interacting with and stabilizing CCND1, leading to the promotion of NSCLC cell proliferation and growth.

Although the association of expression levels of USP5 and CCND1 with the prognosis of NSCLC (LUAD) patients needs to be further addressed after finishing followup in survival data in our study, our analyses of UALCAN and THPA databases demonstrated that both the mRNA and protein levels of USP5, but only protein level of CCND1, show an inverse correlation with good prognosis of LUAD patients. These data reveal that USP5 may be a potential prognosis biomarker for NSCLC and USP5CCND1 axis is implicated in the malignant progression and poor prognosis of NSCLC. It is well known that dysregulated proliferation and metastasis are hallmarks of cancer cells $(33,34)$, which is accountable for malignant features and poor prognosis of cancer patients. USP5 
has been found to promote the epithelial-mesenchymal transition (EMT) and metastasis in NSCLC cells (35). Our cell model data together with Xue's data (35) have clarified that USP5 promotes the proliferation and metastatic malignant biological behaviors of NSCLC cells, thus providing a plausible explanation for the contribution of high level of USP5 to poor prognosis of NSCLC.

As a member of the USP family, USP5 has been found to cleave both linear and branched ubiquitin polymers, and regulates protein ubiquitination, stability, and function $(24,36)$. In line with these functions, a myriad of proteins have been assigned to USP5 as its deubiquitinating substrates, including Forkhead box protein M1 (FoxM1) and signal transducer and activator of transcription 3 (STAT3) in pancreatic cancer $(37,38)$, c-Maf in multiple myeloma $(26,39)$, Tu translation elongation factor (TUFM) in colorectal cancer (40), Slug in hepatocellular carcinoma (41), histone deacetylase 2 (HDAC2) in ovarian cancer (42), $\mathrm{HuR}$ in bladder urothelial carcinoma (43), and v-myc myelocytomatosis viral-related oncogene, neuroblastomaderived (avian) (MYCN) in neuroblastoma (44). More recently, USP5 has been shown to deubiquitinate phosphorylated methyltransferase-like 3 (METTL3) and consequently stabilize it in extracellular signal-regulated kinase (ERK)-activated human cancer cells, thereby contributing to tumorigenesis (45). Accumulating evidence suggests that USP5 exhibits tumor-promoting effects in various tumor types via deubiquitinating and stabilizing oncoproteins (24).

The overexpression of the cell cycle regulator CCND1 as a result of gene amplification or post-transcriptional mechanisms is extremely common in NSCLC, and it represents a key driver marker of NSCLC progression and a potential therapeutic target $(9,10)$. Moreover, CCND1 is associated with the OS rate of patients with lung cancer $(10,11)$. CCND1 is well controlled in cells and dysregulation of its stability might contribute to altered proliferation and survival of various cancer cells. Therefore, a good understanding of CCND1 modulation will help to inform the treatment of NSCLC. Previous studies have shown that USP5 increases the expression of CCND1 in pancreatic cancer (22) and NSCLC (23). Research has also shown that CCND1 is a substrate of USP family members such as USP2a, USP10, and USP22 for the deubiquitination and stabilization of CCND1 (19-21). It has been well demonstrated that some important proteins might have more deubiquitinases to modulate its function, especially in specific biological or pathophysiological contexts. In the present study, we thus set out to examine whether USP5 upregulates CCND1 in NSCLC cells through its deubiquitinase activity.

As shown in our study, the level of USP5 in NSCLC was positively correlated with the expression level of CCND1 protein, which was consistent with the finding of previous reports that ectopic expression of USP 5 results in an increase of CCND1 $(22,23)$. Further, we found that USP5 could physically interact with and deubiquitinate CCND1, thereby stabilizing the protein level of CCND1 and prolonging its half-life. Although the binding sites of USP5 and CCND1 need to be established, our present results clarify that CCND1 is a bona-fide substrate for USP5, and we have deciphered a novel deubiquitinating mechanism for USP5-mediated upregulation of CCND1. Our future work will also focus on whether the USP5-directed deubiquitination of CCND1 is dependent on GSK-3 $\beta$ mediated phosphorylation of CCND1 at T286 (16). Notably, USP22 has been identified as a deubiquitinase of CCND1 in NSCLC (21). Therefore, the contributions of USP5 and USP22 to the deubiquitination and stabilization of CCND1 protein in NSCLC still need to be further addressed. Nonetheless, our data showed that genetic manipulation of USP5 alone could modulate the CCND1 protein level in NSCLC cells in which USP22 was highly expressed, indicating that two members of the USP family, USP5 and USP22, can exert an additional effect in the stabilization of CCND1 via similar or distinct mechanisms. Previous studies have also found that upregulating USP5 facilitates the proliferation, EMT, and metastasis of NSCLC cells through the stabilization of $\beta$-catenin (23) and the activation of $\beta$-catenin signaling $(23,35)$. It is well known that the transcription of CCND1, a classic downstream target of $\beta$-catenin, can be induced by the Wnt- $\beta$-catenin signaling pathway $(46,47)$. These findings suggest that USP5 may also upregulate CCND1 in NSCLC cells at the transcriptional level via activating Wnt- $\beta$-catenin signaling through the stabilization of $\beta$-catenin. In any case, posttranslational regulation of CCND1 stability by USP 5 to upregulate CCND1 protein is of utmost importance to USP5-elicited NSCLC progression.

Accumulating evidence implicates the ubiquitin proteasome system as an extremely important player in numerous types of cancer (48). In current clinical practice, proteasomal inhibitors have achieved accumulating success in the treatment of human cancers, especially multiple myeloma (49). There are also some clinical trials currently underway for the proteasome inhibitor bortezomib as 
a treatment for NSCLC (50-52). However, due to the indispensability of proteasomes in maintaining important cellular and biological activities, attention is increasingly being paid to upstream events, especially in the regulation of protein ubiquitination (49). Due to their diversity and substrate specificity, deubiquitinases stand out as one of the most promising targets (32). In fact, many deubiquitinase inhibitors have been developed and tested in laboratory and clinical trials for the treatment of various cancers (31). Therefore, we were interested in evaluating the therapeutic potential of deubiquitinase inhibitors such as WP1130 and G9, which are small molecule compounds with activity against USP5, in the treatment of NSCLC. In the present study, we found that both WP1130 and G9 displayed marked activity in downregulating CCND1 protein and suppressing NSCLC cell proliferation, which was consistent with the inhibitory effect elicited by the knockdown of USP5 expression. Furthermore, inhibition of USP5 activity with G9 produced remarkable anti-NSCLC activity in vivo and was well tolerated in the nude mouse NSCLC xenograft model. Our observation indicated that pharmacological inhibition of USP5 activity might be a potential treatment modality for NSCLC. However, human clinical trials are needed to further test the safety and efficacy of this treatment approach. If the treatment modality is approved for clinical practice, USP5 may be a potential biomarker for predicating efficacy of USP5-targeted therapy in NSCLC. Treatment with USP5 inhibitor would produce therapeutic benefits for NSCLC patients those have high USP5 expression or activity.

So far, the upstream regulatory mechanism for USP5 expression is little reported. It has been shown that USP5 is a putative target of miR-125a; miR-125a inhibits the malignancy via suppressing the expression of USP 5 in multiple myeloma (53). It has also been shown that mesenchymal stem cells-derived exosome delivers miR-23a$3 p$ into lung epithelial cells and blocks lipopolysaccharide (LPS)-induced acute lung injury via downregulating USP5 (a target of miR-23a-3p) and inhibiting nuclear factor- $\mathrm{KB}$ (NF$\kappa \mathrm{B})$ and hedgehog signaling pathways (54). Interestingly, circle RNA circHECTD1 as a competing endogenous RNA (ceRNA) promotes gastric cancer progression via upregulating USP5 through sponging miR-1256 (55). These evidence implies that USP5 expression can be regulated via a epigenetic post-transcriptional mechanism. Therefore, whether the expression alteration of non-coding RNAs (ncRNAs) is involved in upregulation of USP5 and activation of USP5-CCND1 signaling axis in our NSCLC model needs further investigation in future work. Additionally, CCND1directed $\mathrm{CDK} 4 / 6$ activation and $\mathrm{Rb}$ inactivation may release E2 factor (E2F) transcriptional factor and then transactivate a panel of its downstream genes to accelerate cell cycle progression $(6,7)$. Thus, the E2F-modulated downstream targets elicited by USP5-CCND1-CDK4/6-Rb signaling activation in our NSCLC model also need to be determined in future, which would strengthen our conclusion.

In conclusion, this study has identified that CCND1 is a novel substrate of USP5. By decreasing CCND1 ubiquitination, USP5 stabilizes and upregulates its protein level and consequently promotes NSCLC proliferation. In contrast, genetic or chemical inhibition of USP5 leads to CCND1 degradation, thereby resulting in decreased NSCLC cell proliferation and tumor growth. Thus, targeting of the USP5/CCND1 axis is a novel therapeutic target for NSCLC.

\section{Acknowledgments}

The authors appreciate the academic support from AME Lung Cancer Collaborative Group.

Funding: This work was partly supported by the National Natural Science Foundation of China (\#81672934 to JZ, \#81972841 to XM, and \#81770154 to XM), by Guangzhou Municipal Science and Technology Project (\#202002030059 to XM).

\section{Footnote}

Reporting Checklist: The authors have completed the ARRIVE reporting checklist. Available at https://dx.doi. org/10.21037/tlcr-21-767

Data Sharing Statement: Available at https://dx.doi. org/10.21037/tlcr-21-767

Conflicts of Interest: All authors have completed the ICMJE uniform disclosure form (available at https://dx.doi. org/10.21037/tlcr-21-767). The authors have no conflicts of interest to declare.

Ethical Statement: The authors are accountable for all aspects of the work in ensuring that questions related to the accuracy or integrity of any part of the work are appropriately investigated and resolved. All animal experiments were reviewed and approved by the Review Board of Animal Care and Use of Soochow University 
(Institutional Review Board No. 202008A619), and were performed in compliance with its institutional guidelines for the care and use of laboratory animals. The human study has been conducted in accordance with the Declaration of Helsinki and approved guidelines. The research using human specimens was approved by the Ethics Committee of the First Affiliated Hospital of Soochow University (Institutional Review Board no. 2021-036). Informed consent was taken from all the patients.

Open Access Statement: This is an Open Access article distributed in accordance with the Creative Commons Attribution-NonCommercial-NoDerivs 4.0 International License (CC BY-NC-ND 4.0), which permits the noncommercial replication and distribution of the article with the strict proviso that no changes or edits are made and the original work is properly cited (including links to both the formal publication through the relevant DOI and the license). See: https://creativecommons.org/licenses/by-nc-nd/4.0/.

\section{References}

1. Bray F, Ferlay J, Soerjomataram I, et al. Global cancer statistics 2018: GLOBOCAN estimates of incidence and mortality worldwide for 36 cancers in 185 countries. CA Cancer J Clin 2018;68:394-424.

2. Ramalingam SS, Owonikoko TK, Khuri FR. Lung cancer: New biological insights and recent therapeutic advances. CA Cancer J Clin 2011;61:91-112.

3. Travis WD, Brambilla E, Riely GJ. New pathologic classification of lung cancer: relevance for clinical practice and clinical trials. J Clin Oncol 2013;31:992-1001.

4. Gridelli C, Rossi A, Carbone DP, et al. Non-small-cell lung cancer. Nat Rev Dis Primers 2015;1:15009.

5. Howlader N, Forjaz G, Mooradian MJ, et al. The Effect of Advances in Lung-Cancer Treatment on Population Mortality. N Engl J Med 2020;383:640-9.

6. Massagué J. G1 cell-cycle control and cancer. Nature 2004;432:298-306.

7. Kastan MB, Bartek J. Cell-cycle checkpoints and cancer. Nature 2004;432:316-23.

8. Wu Y, Zhang Y, Pi H, et al. Current Therapeutic Progress of CDK4/6 Inhibitors in Breast Cancer. Cancer Manag Res 2020;12:3477-87.

9. Wikman H, Kettunen E. Regulation of the G1/S phase of the cell cycle and alterations in the RB pathway in human lung cancer. Expert Rev Anticancer Ther 2006;6:515-30.

10. Gautschi O, Ratschiller D, Gugger M, et al. Cyclin D1 in non-small cell lung cancer: a key driver of malignant transformation. Lung Cancer 2007;55:1-14.

11. Musgrove EA, Caldon CE, Barraclough J, et al. Cyclin $\mathrm{D}$ as a therapeutic target in cancer. Nat Rev Cancer 2011;11:558-72.

12. Yang $\mathrm{Y}, \mathrm{Lu} \mathrm{T}, \mathrm{Li} Z$, et al. FGFR1 regulates proliferation and metastasis by targeting CCND1 in FGFR1 amplified lung cancer. Cell Adh Migr 2020;14:82-95.

13. Liu B, Chen D, Chen S, et al. Transcriptional activation of cyclin D1 via HER2/HER3 contributes to EGFR-

TKI resistance in lung cancer. Biochem Pharmacol 2020;178:114095.

14. Li R, An SJ, Chen ZH, et al. Expression of cyclin D1 splice variants is differentially associated with outcome in non-small cell lung cancer patients. Hum Pathol 2008;39:1792-801.

15. Qie S, Diehl JA. Cyclin D1, cancer progression, and opportunities in cancer treatment. J Mol Med (Berl) 2016;94:1313-26.

16. Diehl JA, Cheng M, Roussel MF, et al. Glycogen synthase kinase-3beta regulates cyclin D1 proteolysis and subcellular localization. Genes Dev 1998;12:3499-511.

17. Maeda I, Ohta T, Koizumi H, et al. In vitro ubiquitination of cyclin D1 by ROC1-CUL1 and ROC1-CUL3. FEBS Lett 2001;494:181-5.

18. Lee EK, Diehl JA. SCFs in the new millennium. Oncogene 2014;33:2011-8.

19. Shan J, Zhao W, Gu W. Suppression of cancer cell growth by promoting cyclin D1 degradation. Mol Cell 2009;36:469-76.

20. Sun T, Xu YJ, Jiang SY, et al. Suppression of the USP10/ CCND1 axis induces glioblastoma cell apoptosis. Acta Pharmacol Sin 2021;42:1338-46.

21. Gennaro VJ, Stanek TJ, Peck AR, et al. Control of CCND1 ubiquitylation by the catalytic SAGA subunit USP22 is essential for cell cycle progression through G1 in cancer cells. Proc Natl Acad Sci U S A 2018;115:E9298-307.

22. Kaistha BP, Krattenmacher A, Fredebohm J, et al. The deubiquitinating enzyme USP5 promotes pancreatic cancer via modulating cell cycle regulators. Oncotarget 2017;8:66215-25.

23. Ma X, Qi W, Pan H, et al. Overexpression of USP5 contributes to tumorigenesis in non-small cell lung cancer via the stabilization of $\beta$-catenin protein. Am J Cancer Res 2018;8:2284-95.

24. Ning F, Xin H, Liu J, et al. Structure and function of USP5: Insight into physiological and pathophysiological 
roles. Pharmacol Res 2020;157:104557.

25. Issaenko OA, Amerik AY. Chalcone-based small-molecule inhibitors attenuate malignant phenotype via targeting deubiquitinating enzymes. Cell Cycle 2012;11:1804-17.

26. Wang S, Juan J, Zhang Z, et al. Inhibition of the deubiquitinase USP5 leads to c-Maf protein degradation and myeloma cell apoptosis. Cell Death Dis 2017;8:e3058.

27. Zhang $Z$, Tong J, Tang $X$, et al. The ubiquitin ligase HERC4 mediates c-Maf ubiquitination and delays the growth of multiple myeloma xenografts in nude mice. Blood 2016;127:1676-86.

28. Bartholomeusz G, Talpaz M, Bornmann W, et al. Degrasyn activates proteasomal-dependent degradation of c-Myc. Cancer Res 2007;67:3912-8.

29. Peterson LF, Sun H, Liu Y, et al. Targeting deubiquitinase activity with a novel small-molecule inhibitor as therapy for B-cell malignancies. Blood 2015;125:3588-97.

30. Xie $\mathrm{P}$, Zhang $\mathrm{M}, \mathrm{He} \mathrm{S}$, et al. The covalent modifier Nedd8 is critical for the activation of Smurf1 ubiquitin ligase in tumorigenesis. Nat Commun 2014;5:3733.

31. Harrigan JA, Jacq X, Martin NM, et al. Deubiquitylating enzymes and drug discovery: emerging opportunities. Nat Rev Drug Discov 2018;17:57-78.

32. Fraile JM, Quesada V, Rodríguez D, et al. Deubiquitinases in cancer: new functions and therapeutic options. Oncogene 2012;31:2373-88.

33. Hanahan D, Weinberg RA. The hallmarks of cancer. Cell 2000;100:57-70.

34. Hanahan D, Weinberg RA. Hallmarks of cancer: the next generation. Cell 2011;144:646-74.

35. Xue S, Wu W, Wang Z, et al. USP5 Promotes Metastasis in Non-Small Cell Lung Cancer by Inducing EpithelialMesenchymal Transition via Wnt/ $\beta$-Catenin Pathway. Front Pharmacol 2020;11:668.

36. Nijman SM, Luna-Vargas MP, Velds A, et al. A genomic and functional inventory of deubiquitinating enzymes. Cell 2005;123:773-86.

37. Li XY, Wu HY, Mao XF, et al. USP5 promotes tumorigenesis and progression of pancreatic cancer by stabilizing FoxM1 protein. Biochem Biophys Res Commun 2017;492:48-54.

38. Lian J, Liu C, Guan X, et al. Ubiquitin specific peptidase 5 enhances STAT3 signaling and promotes migration and invasion in Pancreatic Cancer. J Cancer 2020;11:6802-11.

39. Chen XH, Xu YJ, Wang XG, et al. Mebendazole elicits potent antimyeloma activity by inhibiting the USP5/c-Maf axis. Acta Pharmacol Sin 2019;40:1568-77.

40. Xu X, Huang A, Cui X, et al. Ubiquitin specific peptidase 5 regulates colorectal cancer cell growth by stabilizing Tu translation elongation factor. Theranostics 2019;9:4208-20.

41. Meng J, Ai X, Lei Y, et al. USP5 promotes epithelialmesenchymal transition by stabilizing SLUG in hepatocellular carcinoma. Theranostics 2019;9:573-87.

42. Du Y, Lin J, Zhang R, et al. Ubiquitin specific peptidase 5 promotes ovarian cancer cell proliferation through deubiquitinating HDAC2. Aging (Albany NY) 2019;11:9778-93.

43. Xiao M, Liu J, Xiang L, et al. MAFG-AS1 promotes tumor progression via regulation of the HuR/PTBP1 axis in bladder urothelial carcinoma. Clin Transl Med 2020;10:e241.

44. Cheung BB, Kleynhans A, Mittra R, et al. A novel combination therapy targeting ubiquitin-specific protease 5 in MYCN-driven neuroblastoma. Oncogene 2021;40:2367-81.

45. Sun HL, Zhu AC, Gao Y, et al. Stabilization of ERKPhosphorylated METTL3 by USP5 Increases m6A Methylation. Mol Cell 2020;80:633-647.e7.

46. Zhan T, Rindtorff N, Boutros M. Wnt signaling in cancer. Oncogene 2017;36:1461-73.

47. Anastas JN, Moon RT. WNT signalling pathways as therapeutic targets in cancer. Nat Rev Cancer 2013;13:11-26.

48. D'Arcy P, Linder S. Molecular pathways: translational potential of deubiquitinases as drug targets. Clin Cancer Res 2014;20:3908-14.

49. Antao AM, Tyagi A, Kim KS, et al. Advances in Deubiquitinating Enzyme Inhibition and Applications in Cancer Therapeutics. Cancers (Basel) 2020;12:1579.

50. Mofers A, Pellegrini P, Linder S, et al. Proteasomeassociated deubiquitinases and cancer. Cancer Metastasis Rev 2017;36:635-53.

51. Kontopodis E, Kotsakis A, Kentepozidis N, et al. A phase II, open-label trial of bortezomib (VELCADE(®)) in combination with gemcitabine and cisplatin in patients with locally advanced or metastatic non-small cell lung cancer. Cancer Chemother Pharmacol 2016;77:949-56.

52. Zhao Y, Foster NR, Meyers JP, et al. A phase I/II study of bortezomib in combination with paclitaxel, carboplatin, and concurrent thoracic radiation therapy for non-smallcell lung cancer: North Central Cancer Treatment Group (NCCTG)-N0321. J Thorac Oncol 2015;10:172-80.

53. Wu L, Zhang C, Chu M, et al. miR-125a suppresses malignancy of multiple myeloma by reducing the deubiquitinase USP5. J Cell Biochem 2020;121:642-50. 
54. Xiao K, He W, Guan W, et al. Mesenchymal stem cells reverse EMT process through blocking the activation of $\mathrm{NF}-\mathrm{\kappa B}$ and Hedgehog pathways in LPS-induced acute lung injury. Cell Death Dis 2020;11:863.

Cite this article as: Zhang Z, Cui Z, Xie Z, Li C, Xu C, Guo X, Yu J, Chen T, Facchinetti F, Bohnenberger H, Leong TL, Xie Y, Mao X, Zhao J. Deubiquitinase USP5 promotes non-small cell lung cancer cell proliferation by stabilizing cyclin D1. Transl Lung Cancer Res 2021;10(10):3995-4011. doi: 10.21037/tlcr21-767
55. Cai J, Chen Z, Wang J, et al. circHECTD1 facilitates glutaminolysis to promote gastric cancer progression by targeting miR-1256 and activating $\beta$-catenin/c-Myc signaling. Cell Death Dis 2019;10:576. 